\title{
A Note on Multiple Comparison Procedures for Analysis of Ranked Data
}

\author{
James A. Koziol \\ College of Health, Human Services and Science, Ashford University \\ *Corresponding Author: James.koziol@faculty.ashford.edu
}

Copyright $@ 2013$ Horizon Research Publishing All rights reserved.

\begin{abstract}
Ranked samples typically arise in sensory evaluation studies, in which a group of paneliests independently rank the same set of samples. In this setting, Christensen and colleagues (Journal of Food Science 71:S132-S143, 2006) have proposed a nonparametric procedure for multiple comparisons of the samples, based on the sums of the ranks of the samples, summed across the panelists' rankings. Christensen and colleagues utilized a simulation procedure in order to estimate critical differences between rank sums, at various alpha levels and combinations of numbers of panelists and samples. In this note, exact distribution theory relating to the rank sums is derived, using probability generating functions; and, critical values can be determined through computer algebra.
\end{abstract}

Keywords Sensory Analysis, Friedman Rank Test, Probability Generating Functions, Multiple Comparisons

\section{Introduction}

A common use of rank statistics is to compare samples that have been independently evaluated by a group of panelists. Typically, a number $\mathrm{n}$ of panelists independently examine the same $\mathrm{k}$ samples, and ascribe ranks 1 through $\mathrm{k}$ to the samples; then, the rank sums of each sample, summed across all of the panelists' rankings, are used to evaluate and compare the samples. Friedman's rank test (Friedman, 1937) is an omnibus nonparametric procedure for assessing whether the rank sums of the samples differ significantly. In practice, comparisons among particular samples may be interest, and alternatives to the Friedman test have been proposed for multiple comparisons. Joanes (1985) suggested that inference be based on the largest absolute difference between rank sums, and Newell (1987) and Basker (1988) provided associated tables of critical values. Christensen et al. (2006) noted that the largest absolute difference could be conservative, and instead proposed that pairwise rank sum differences be used for multiple comparisons. Following Joanes (1985), empirical distributions of absolute maximum rank sum differences, and individual rank sum differences, were obtained via simulation studies, and tables were prepared for general use.

To determine the exact distribution of rank sums seems a formidable problem. With $\mathrm{k}$ samples and $\mathrm{n}$ panelists, there are $(\mathrm{k} !)^{\mathrm{n}}$ possible rank configurations, equally likely under the null hypothesis that each of the panelists' $\mathrm{k}$ ! potential rankings is equally likely. Nevertheless, it is feasible to obtain the exact distribution of rank sums, using probability generating functions [e.g., Feller (1968)]. The purpose of this note is to demonstrate the use of probability generating functions for Christensen's multiple comparisons procedure based on individual rank sum differences. Mathematical details are given in the next section, and tables complementing those presented by Christensen et al. (2006) are given in the Results.

\section{Methods}

Let us briefly review probability generating functions. [We refer the interested reader to Feller (1968) for a simple but elegant introduction.] Suppose $\mathrm{X}$ is a discrete random variable taking values on the non-negative integers, $Z_{\geq 0}=\{0,1,2, \ldots\}$, and let $p_{i}$ denote the probability $\operatorname{Prob}(X=i)$, for $\mathrm{i}$ in $Z_{\geq 0}$. Then the probability generating function $\mathrm{G}$ of $\mathrm{X}$ is defined as

$$
G(z)=E\left(z^{X}\right)=\sum_{i=0}^{\infty} p_{i} z^{i}
$$

The probability generating function is quite useful, as it allows determination of many basic properties of X. For example, one can recover the probability mass function of $\mathrm{X}$ by taking derivatives of $\mathrm{G}$ :

$$
p_{i}=\frac{G^{(i)}(0)}{i !}, i=0,1,2,,,
$$

where $G^{(i)}(0)$ denotes the $i^{\text {th }}$ derivative of $G$ with respect to $z$, evaluated at $\mathrm{z}=0$.

We note one other property of probability generating functions, which we will later exploit: If $X_{1}, X_{2}, \ldots, X_{n}$ is a 
sequence of independent random variables with respective probability generating functions $G_{X_{i}}$, then the probability generating function of the sum $\mathrm{S}_{\mathrm{n}}=\mathrm{X}_{1}+\mathrm{X}_{2}+\ldots+\mathrm{X}_{\mathrm{n}}$ is given by

$$
G_{S_{n}}(z)=G_{X_{1}}(z) G_{X_{2}}(z) \ldots G_{X_{n}}(z) .
$$

In the present setting, with 2 or more independent samples, we introduce the bivariate probability generating function. Let $\mathrm{X}=\left(\mathrm{X}_{1}, \mathrm{X}_{2}\right)$ be a discrete random variable taking values in the 2-dimensional non-negative integer lattice $\{0,1,2, \ldots\} \mathrm{x}$ $\{0,1,2, \ldots\}$, and let $\mathrm{p}_{\mathrm{ij}}$ denote the probability $\operatorname{Prob}\left(\mathrm{X}_{1}=\mathrm{i}, \mathrm{X}_{2}=\mathrm{j}\right)$ for $(\mathrm{i}, \mathrm{j})$ in the lattice. Then the probability generating function of $\mathrm{X}$ is defined as

$$
\begin{aligned}
& G(z)=G\left(z_{1}, z_{2}\right) \\
& =E\left(z_{1}^{X_{1}} z_{2}^{X_{2}}\right)=\sum_{i, j=0}^{\infty} p_{i j} z_{1}^{i} z_{2}^{j}
\end{aligned}
$$

Now we apply these notions so as to determine the exact distributions of Christensen's individual rank sums. We have $\mathrm{n}$ panelists independently ranking the same $\mathrm{k}$ samples. Each panelist can effect any of $\mathrm{k}$ ! distinct rankings of the $\mathrm{k}$ samples, and these are taken as equally likely under the null hypothesis of no sample differences. Let us focus on sample 1 and sample 2 for convenience, these representing $X_{1}$ and $\mathrm{X}_{2}$ in the bivariate setting described above. [The samples are exchangeable under the null hypothesis, so we may focus on samples 1 and 2 without any loss of generality.] There are $\mathrm{k}(\mathrm{k}-1)$ possible joint rankings of $\mathrm{X}_{1}$ and $\mathrm{X}_{2}$ by any of the panelists, and these $\mathrm{k}(\mathrm{k}-1)$ joint rankings are equally likely. We outline the steps leading to the determination of the exact probabilities: (i) we first find the bivariate probability generating function for $\mathrm{X}_{1}$ and $\mathrm{X}_{2}$ as described previously; (ii) we raise this probability generating function to the $\mathrm{n}^{\text {th }}$ power, this being the probability generating function for the rank sums of samples 1 and 2, with summation over the $n$ independent panelist evaluations; (iii) we calculate the exact probability distribution for the absolute value of the difference in rank sums by examination of the coefficients of the power series expansion of the rank sum probability generating function.
Each of these steps is conceptually straightforward, as we are merely utilizing known properties of probability generating functions. In practice, implementation of these steps can be enormously complicated, but, as pointed out be van de Wiel et al. (1998), can be readily facilitated with computer algebra systems such as Mathematica (Wolfram Research, Inc.). Mathematica is a powerful program, particularly adept at symbolic calculations, and we utilize Mathematica for each of the steps outlined previously. In the Appendix we give a list of Mathematica functions and commands that follow the above rubric for finding the exact distributions of Christensen's statistics, absolute values of differences in rank sums.

\section{Results}

For completeness, we extend the multiple comparisons tables of Christensen et al. (2006), their Tables 5, 6, and 7, to numbers of panelists 5 through 19 , while maintaining numbers of samples 3 through 12, and alpha levels [P values] $0.10,0.05$, and 0.01 . The critical values are given in Table 1 below. These numbers were extracted from the exact distributions of the absolute values of rank sum differences.

We must emphasize that the rank sums have a discrete distribution, hence critical values may well be conservative. We illustrate this point with a small configuration, namely, 3 samples, 2 panelists $[t=3, b=2$, following the notational conventions introduced in the Appendix]. In this setting, there are a total of 5 possible values for the absolute differences in rank sums from any two samples:

\begin{tabular}{|c|c|c|c|c|c|}
\hline $\begin{array}{c}\text { Absolute } \\
\text { Difference }\end{array}$ & 0 & 1 & 2 & 3 & 4 \\
\hline Probability & .278 & .222 & .222 & .222 & .056 \\
\hline
\end{tabular}

[The computationally inclined reader might verify that the exact probabilities are 5/18, 2/9, 2/9, 2/9, and $1 / 18$ respectively.] It is immediately clear that a rank sum of 4 would be "significant" at the alpha $=.10$ level, though the substantial difference between the However, there is no critical value for alpha levels 0.05 or 0.01 here. And, one might argue that an achieved significance level is more informative than a prespecified alpha level, given discreteness. 
Table 1. Critical Values of Absolute Differences Between Rank Sums

\begin{tabular}{|c|c|c|c|c|c|c|c|c|c|c|}
\hline \multirow{3}{*}{$\begin{array}{c}\text { Number } \\
\text { of Panelists }\end{array}$} & & & \multicolumn{7}{|c|}{ A. Critical values of absolute differences between rank sums, alpha $=\mathbf{0 . 1 0}$} & \\
\hline & & & & & \multicolumn{2}{|c|}{ Number of Samples } & \multirow[b]{3}{*}{9} & \multirow[b]{3}{*}{10} & \multirow[b]{3}{*}{11} & \multirow[b]{3}{*}{12} \\
\hline & & & & & & & & & & \\
\hline & 3 & 4 & 5 & 6 & 7 & 8 & & & & \\
\hline 5 & 5 & 7 & 8 & 10 & 11 & 13 & 14 & 16 & 17 & 19 \\
\hline 6 & 6 & 7 & 9 & 11 & 12 & 14 & 16 & 17 & 19 & 21 \\
\hline 7 & 6 & 8 & 10 & 12 & 13 & 15 & 17 & 19 & 20 & 22 \\
\hline 8 & 7 & 8 & 10 & 12 & 14 & 16 & 18 & 20 & 22 & 24 \\
\hline 9 & 7 & 9 & 11 & 13 & 15 & 17 & 19 & 21 & 23 & 25 \\
\hline 10 & 7 & 10 & 12 & 14 & 16 & 18 & 20 & 22 & 24 & 27 \\
\hline 11 & 8 & 10 & 12 & 14 & 17 & 19 & 21 & 23 & 26 & 28 \\
\hline 12 & 8 & 10 & 13 & 15 & 17 & 20 & 22 & 24 & 27 & 29 \\
\hline 13 & 8 & 11 & 13 & 16 & 18 & 21 & 23 & 25 & 28 & 30 \\
\hline 14 & 9 & 11 & 14 & 16 & 19 & 21 & 24 & 26 & 29 & 31 \\
\hline 15 & 9 & 12 & 14 & 17 & 19 & 22 & 25 & 27 & 30 & 33 \\
\hline 16 & 9 & 12 & 15 & 17 & 20 & 23 & 25 & 28 & 31 & 34 \\
\hline 17 & 10 & 12 & 15 & 18 & 21 & 24 & 26 & 29 & 32 & 35 \\
\hline 18 & 10 & 13 & 16 & 18 & 21 & 24 & 27 & 30 & 33 & 36 \\
\hline \multirow[t]{2}{*}{19} & 10 & 13 & 16 & 19 & 22 & 25 & 28 & 31 & 34 & 37 \\
\hline & & & \multicolumn{7}{|c|}{ B. Critical values of absolute differences between rank sums, alpha $=0.05$} & \\
\hline & & & & & & & & & & \\
\hline \multirow{3}{*}{$\begin{array}{l}\text { Number } \\
\text { of Panelists }\end{array}$} & & & & & \multicolumn{2}{|c|}{ Number of Samples } & & & & \\
\hline & & & & & & & & & & \\
\hline & 3 & 4 & 5 & 6 & 7 & 8 & 9 & 10 & 11 & 12 \\
\hline 5 & 6 & 8 & 10 & 11 & 13 & 15 & 17 & 19 & 20 & 22 \\
\hline 6 & 7 & 9 & 11 & 13 & 15 & 17 & 18 & 20 & 22 & 24 \\
\hline 7 & 7 & 9 & 12 & 14 & 16 & 18 & 20 & 22 & 24 & 26 \\
\hline 8 & 8 & 10 & 12 & 15 & 17 & 19 & 21 & 24 & 26 & 28 \\
\hline 9 & 8 & 11 & 13 & 15 & 18 & 20 & 23 & 25 & 27 & 30 \\
\hline 10 & 9 & 11 & 14 & 16 & 19 & 21 & 24 & 26 & 29 & 32 \\
\hline 11 & 9 & 12 & 14 & 17 & 20 & 22 & 25 & 28 & 30 & 33 \\
\hline 12 & 10 & 12 & 15 & 18 & 21 & 23 & 26 & 29 & 32 & 35 \\
\hline 13 & 10 & 13 & 16 & 19 & 22 & 24 & 27 & 30 & 33 & 36 \\
\hline 14 & 10 & 13 & 16 & 19 & 22 & 25 & 28 & 31 & 34 & 37 \\
\hline 15 & 11 & 14 & 17 & 20 & 23 & 26 & 29 & 32 & 36 & 39 \\
\hline 16 & 11 & 14 & 17 & 21 & 24 & 27 & 30 & 33 & 37 & 40 \\
\hline 17 & 11 & 15 & 18 & 21 & 25 & 28 & 31 & 35 & 38 & 41 \\
\hline 18 & 12 & 15 & 19 & 22 & 25 & 29 & 32 & 36 & 39 & 42 \\
\hline \multirow[t]{2}{*}{19} & 12 & 16 & 19 & 23 & 26 & 30 & 33 & 37 & 40 & 43 \\
\hline & & & \multicolumn{7}{|c|}{ C. Critical values of absolute differences between rank sums, alpha $=0.01$} & \\
\hline & & & & & & & & & & \\
\hline \multirow{3}{*}{$\begin{array}{l}\text { Number } \\
\text { of Panelists }\end{array}$} & & & & & Number & oles & & & & \\
\hline & & & & & & & & & & \\
\hline & 3 & 4 & 5 & 6 & 7 & 8 & 9 & 10 & 11 & 12 \\
\hline 5 & 8 & 10 & 12 & 15 & 17 & 19 & 22 & 24 & 26 & 29 \\
\hline 6 & 9 & 11 & 14 & 16 & 19 & 21 & 24 & 26 & 29 & 31 \\
\hline 7 & 9 & 12 & 15 & 18 & 20 & 23 & 26 & 29 & 31 & 34 \\
\hline 8 & 9 & 12 & 15 & 18 & 20 & 23 & 26 & 29 & 31 & 34 \\
\hline 9 & 11 & 14 & 17 & 20 & 23 & 26 & 29 & 33 & 36 & 39 \\
\hline 10 & 11 & 15 & 18 & 21 & 24 & 28 & 31 & 34 & 38 & 41 \\
\hline 11 & 12 & 15 & 19 & 22 & 26 & 29 & 33 & 36 & 40 & 43 \\
\hline 12 & 12 & 16 & 20 & 23 & 27 & 31 & 34 & 38 & 41 & 45 \\
\hline 13 & 13 & 17 & 20 & 24 & 28 & 32 & 36 & 39 & 43 & 47 \\
\hline 14 & 13 & 17 & 21 & 25 & 29 & 33 & 37 & 41 & 45 & 49 \\
\hline 15 & 14 & 18 & 22 & 26 & 30 & 34 & 38 & 42 & 46 & 50 \\
\hline 16 & 14 & 19 & 23 & 27 & 31 & 35 & 40 & 44 & 48 & 52 \\
\hline 17 & 15 & 19 & 23 & 28 & 32 & 36 & 41 & 45 & 49 & 54 \\
\hline 18 & 15 & 20 & 24 & 29 & 33 & 38 & 42 & 46 & 51 & 55 \\
\hline 19 & 16 & 20 & 25 & 29 & 34 & 39 & 43 & 48 & 52 & 57 \\
\hline
\end{tabular}




\section{Final Remarks}

Christensen et al. (2006) have cogently argued that absolute differences in pairwise rank sums are preferable to maximum absolute differences for multiple comparisons with ranked data.The exact distributions of Christensen's pairwise rank sums can be determined, through computer algebra, and we have implemented these calculations in Mathematica. We emphasize that the exact distributions are discrete, so that testing at pre-specified levels of significance may well introduce conservativeness in inferential procedures. In practice, it might be preferable to report achieved levels of significance rather than testing at a prespecifiedalpha level; this is readily accomplished, since exact probabilities of absolute rank sum differences are easily obtained by the methods outlined in this note.

\section{Appendix}

Motivated by van de Wiel et al. (1998, 2004), we give a number of functions in Mathematica that can be used to determine the exact distributions of Christensen's absolute values of pairwise rank sum differences. In these functions, $t$ will denote the number of samples (treatments), and b will denote the number of panelists (blocks). The semicolon at the end of each line of code is Mathematica syntax to suppress printing. [These functions were derived and implemented in Mathematica 8.]

We start with

$\operatorname{pgf}[\mathrm{t}]:=\operatorname{pgf}[\mathrm{t}]=$ Expand [

$\operatorname{Sum}\left[\left(x[1]^{\wedge} \mathrm{i}\right) *\left(x[2]^{\wedge} \mathrm{j}\right)+\left(x[1]^{\wedge} \mathrm{j}\right) *\left(x[2]^{\wedge} \mathrm{i}\right),\{\mathrm{i}, 1, \mathrm{t}-1\},\{\mathrm{j}, \mathrm{i}+\right.$ $1, \mathrm{t}\}]]$;

pgf[t] calculates the bivariate generating function of $X_{1}$ and $X_{2}[$ or, $x[1]$ and $x[2]$ in Mathematica notation] with $t$ samples. Notice that we have not included the normalizing constant $(\mathrm{t}(\mathrm{t}-1))^{-1}$ in the pgf statement: we will defer normalization to the end.

Next,

FriedmanGF[t_, b_] := Expand $\left[\operatorname{pgf}[\mathrm{t}]^{\wedge} \mathrm{b}\right]$;

FriedmanGF[t,b] is the bivariate generating function of the rank sums of $X_{1}$ and $X_{2}$ (without normalization yet), with $\mathrm{t}$ samples and $\mathrm{b}$ panelists. Since the panelists produce independent rankings, we invoke the property that the generating function of the sum of independent variables is the product of the individual generating functions.

We introduce a helper function

$\mathrm{ff}\left[\left(\mathrm{d}_{-}: 1\right)^{*} \mathrm{x}[1]^{\wedge}\left(\mathrm{a}_{-}: 1\right)^{*} \mathrm{x}[2]^{\wedge}\left(\mathrm{b}_{-}: 1\right)\right]:=\mathrm{d}^{*} \mathrm{tt}^{\wedge}(\operatorname{Abs}[\mathrm{b}-\mathrm{a}])$;

FriedmanGF is the generating function for the rank sums of $\mathrm{X}_{1}$ and $\mathrm{X}_{2}$, but Christensen bases inference on the absolute values of the rank sum differences. Hence we need to construct a univariate probability generating function for the absolute values of the rank sum differences. The helper function ff takes an arbitrary term in the FriedmanGF expansion, determines the absolute value of the rank sum difference from that term (this is Abs[b-a]) as well as the relative frequency of that absolute rank sum difference (this is the coefficient $\mathrm{d}$ in the FriedmanGF expansion), and rewrites the term appropriately.

We use ff in the following function:

FriedmanIndivProbs[t_, $\left.\mathrm{b}_{-}\right]:=\operatorname{Module}[\{$ temp $\}$, temp $=$ CoefficientList $[$ Map[ff, FriedmanGF[t, b]], tt]; temp $=$ temp/Total[temp] ];

Given $\mathrm{t}$ and $\mathrm{b}$, FriedmanIndivProbs[t,b] will return an ordered list of length $(\mathrm{t}-1) * \mathrm{~b}+1$. This list contains the individual probabilities that the absolute rank sum difference is $0,1, \ldots,(\mathrm{t}-1)^{*} \mathrm{~b}$. [The probabilities will sum to 1 , since we have normalized the entries with the Total[] function.]

We may obtain a list of cumulative probabilities with the function

FriedmanCumProbs[t_, b_] := Drop[FoldList[Plus, 0, FriedmanIndivProbs[t,b]],1];

Exceedance probabilities, Prob( absolute rank sum difference $>\mathrm{k} \mid \mathrm{t}, \mathrm{b})$ are given with

FriedmanRightTail[t_, b_, k_]:=

$\mathrm{N}[1$ - Part[FriedmanCumProbs[t, b], k+1] ];

In practice, then, observed values of absolute rank sum differences can be assessed via individual probabilities, cumulative probabilities, or exceedance probabilities. [We caution that indices in Mathematica lists begin with 1 , but our individual and cumulative probability lists begin with probabilities corresponding to absolute rank sum differences $=0$.]

Christensen has provided tables of critical values of absolute differences of rank sums at alpha levels .10, .05, and .01, and these may be obtained with the function

FriedmanRightCriticalValue[t_, b_, alpha_] :=

Module $[\{$ temp $\}, \quad$ temp $=$ Length[Select $[1$

FriedmanCumProbs[t, b], \# < alpha \&]]; If[1 < temp, $(\mathrm{t}-1) * \mathrm{~b}+1$ - temp, "*"]]

For given $t, b$, and prespecified alpha level (e.g., .10), this right critical value function will give the minimal value such that the probability of the absolute rank sum difference exceeding this value will be $\leq$ alpha.

\section{REFERENCES}

[1] Basker D. 1988. Critical values of differences among ranked sums for multiple comparisons. Food Technol 42:79-84.

[2] Christensen ZT, Ogden LV, Dunn ML, Eggett DL. 2006. Multiple comparison procedures for analysis of paired data. $\mathrm{J}$ Food Sci 71:S132-S143.

[3] Feller W. An Introduction to Probability Theory and its Applications, Vol. 1, $3^{\text {rd }}$ Edition. John Wiley: New York.

[4] Friedman M. 1937.The use of ranks to avoid the assumption of normality implicit in the analysis of variance. J Amer Statist Assoc 32: 675-701.

[5] Joanes DA. 1985. On a rank sum test due to Kramer. J Food Sci 50:1442-1444.

[6] Newell GJ, McFarlane JD. 1987. Expanded tables for 
multiple comparison procedures in the analysis of ranked data. J Food Sci 52:1721-1725.

[7] van de Wiel MA. 2004. Exact null distributions of quadratic distribution-free statistics for two-way classification. J Statist
Plann Inference 120:29-40.

[8] van de Wiel MA, Di Bucchianico A, van der Laan P. 1998. Symbolic computation and exact distributions of nonparametric rank statistics. The Statistician 48:507-516. 